\title{
Editorial
}

\section{The Intensive Care Unit in the "COVID-19 Era"}

\author{
Sanjay Orathi Patangi ${ }^{1}$ Muralidhar Kanchi \\ ${ }^{1}$ Department of Adult Cardiac Intensive Care, Narayana Institute \\ of Cardiac Sciences, Narayana Health City, Bangalore, Karnataka, \\ India \\ ${ }^{2}$ Department of Anaesthesia and Intensive Care, Narayana Institute \\ of Cardiac Sciences, Narayana Health City, Bangalore, Karnataka, \\ India
}

J Card Crit Care:2020;4:75-78

"Intensive care therapy in these testing times is not about waiting for the storm to pass but learning how to dance in the rain.".

We are in the midst of a global pandemic caused by coronavirus disease 2019 (COVID-19). Thirty-three percent of infected patients with coronavirus manifest severe acute respiratory illness and invariably need intensive care unit (ICU) admission. ${ }^{1}$ ICU preparedness for this pandemic poses unique challenges for the ICU requirements that include infrastructure, staffing, infection control, and in-depth knowledge of management. Appropriate management and minimizing risks to health care workers (HCW) should feature high on institutional protocols to protect and have adequately trained front-line workers. These protocols include infection control policies, dissemination of information to HCW, resuscitation and code blue protocols, advanced ICU services including extracorporeal membrane oxygenation (ECMO) along with psychological, and emotional support services to prevent burn-out of HCW in these potentially high-risk areas. ${ }^{2}$ The Society of Critical Care Medicine has formed an ICU preparedness checklist that encompasses review of preparedness, logistical capacity, triage, staffing capacity, equipment requirements along with channels of communication to safely handle these patients and mitigate infection spread among HCW. ${ }^{3}$

Novel respiratory viruses such as the coronavirus require effective preventive strategies to maintain staff safety. Use of N95 masks, personal protective equipment (PPE), and negative pressure rooms is recommended when aerosolizing procedures such as endotracheal intubation, bronchoscopy, and tracheostomy are performed. ${ }^{4}$ Ethical considerations and guidance for providing cardiopulmonary resuscitation (CPR) outline three recommendations that encompass goals of CPR, do not actively resuscitate (DNAR) status, and safety of HCW during CPR. ${ }^{5}$ Termination of resuscitative efforts and guidance on postresuscitative care have been extensively debated. ${ }^{6}$ In this editorial, we highlight the clinical modalities of this novel disease along with logistical solutions to handle the crisis situation that has arisen in its wake.

\section{Current Clinical Modalities}

High-flow nasal cannula oxygenation and noninvasive ventilation (NIV) form the initial modalities of treatment for hypoxia associated with mild-to-moderate respiratory illness in COVID-19 infection. NIV provides positive end-expiratory pressure, decreases work of breathing, and may reduce incidence of intubation. ${ }^{7}$ However, there is insufficient evidence to prove the efficacy of one over the other. Once endotracheally intubated, lung protective strategies such as low tidal volume: 6 to $8 \mathrm{~mL} / \mathrm{kg}$, limited plateau pressure $<30 \mathrm{~cm}$ $\mathrm{H}_{2} \mathrm{O}$, and a driving pressure $<15 \mathrm{~cm} \mathrm{H}_{2} \mathrm{O}$ are recommended especially if the respiratory failure falls into the $\mathrm{H}$ category. High-positive end-expiratory pressure has not been found to be successful in managing COVID-19 patients. ${ }^{7}$ The recommended practice during bronchoscopy includes setting the ventilatory to stand-by mode, briefly clamping the artificial airway, quick insertion of the bronchoscope prior to restoration of ventilation. ${ }^{8}$ Prone ventilation improves lung

\author{
Address for correspondence \\ Muralidhar Kanchi, MD, FIACTA, FICA, \\ MBA, FASE, Department of Anaesthesia \\ and Intensive Care, Narayana Institute of \\ Cardiac Sciences, Narayana Health City, \\ \#258/A, Bommasandra Industrial Area, \\ Anekal Taluk, Bangalore 560 099, \\ Karnataka, India (e-mail: \\ muralidhar.kanchi.dr@narayanahealth.org,/ \\ kanchirulestheworld@gmail.com).
}

DOI https://doi.org/ $10.1055 / \mathrm{s}-0040-1718649$ ISSN 2457-0206.

\footnotetext{
(c) 2020. Official Publication of The Simulation Society (TSS), accredited by International Society of Cardiovascular Ultrasound (ISCU).

This is an open access article published by Thieme under the terms of the Creative Commons Attribution-NonDerivative-NonCommercial-License, permitting copying and reproduction so long as the original work is given appropriate credit. Contents may not be used for commercial purposes, or adapted, remixed, transformed or built upon. (https://creativecommons.org/licenses/by-nc-nd/4.0/) Thieme Medical and Scientific Publishers Pvt. Ltd., A-12, 2nd Floor, Sector 2, Noida-201301 UP, India
} 
homogeneity and early proning is recommended. ${ }^{7}$ If optimization of ventilatory management and prone ventilation strategies fail, venovenous (VV) ECMO may be considered. VV-ECMO is a well-established therapy for viral pneumonia and acute respiratory distress syndrome. ${ }^{9}$ ECMO increases resource utilization on the backdrop of uncertainty related to reversibility of the primary cause. To achieve maximum benefit, it should be instituted early to provide oxygenation, carbon dioxide washout, and ventilator-associated lung injury. ${ }^{\text {? }}$

The role of antiviral drugs including ribavirin and remdesivir has not been found to provide improvement in clinical outcomes in patients suffering from severe COVID-19. ${ }^{10} \mathrm{Few}$ reports ${ }^{7}$ have shown benefits of corticosteroids when used early, contrary to reports of high mortality when used after day 14.

Disseminated intravascular coagulation is commonly found in COVID-19 patients. Its proposed mechanism includes effects of virus on vascular endothelium and cytokine mediated coagulation cascade interference. Thrombocytopenia is an independent marker of poor prognosis in COVID-19 patients. ${ }^{11}$ Autopsy reports on COVID-19 patients have revealed transparent thrombus in alveoli, myocardium, portal area, and renal tubular cells indicating fibrinolysis inhibition. ${ }^{7}$ Hypercoaguable state following COVID-19 infections responds well to heparin. Hence, low molecular weight heparin or unfractionated heparin is the anticoagulant of choice and their usage has shown improved survival. ${ }^{12}$

Acute kidney injury (AKI) in COVID-19 patients has been attributed to the effect of viral load on target cells in the kidney, immune-mediated damage, disease-related prerenal factors, and drug-related nephrotoxicity. AKI is an independent risk factor for poor prognosis. ${ }^{13}$ The indications for renal replacement therapy include fluid management, oliguria and metabolic management and hypernatremia. ${ }^{7}$ Convalescent plasma lowers upper respiratory tract viral load, decreases serum cytokine levels, and causes passive immunization but there is no clear evidence to state this mode of therapy is efficacious. $^{\text {? }}$

Early nutrition therapy within 48 hours of admission and preferential use of enteral mode of nutrition is recommended with a moderately hypocaloric diet. The use of high energy enteral nutrition should be weighed against excessive volume load. However, enteral nutrition should be delayed in COVID-19 patients who present with sever shock states, metabolic acidosis, gastrointestinal bleed, and abdominal compartment syndrome. ${ }^{14}$

Elevated myocardial biomarkers such as troponin and creatine kinase have been observed in COVID-19 patients indicative of myocardial injury with poor prognosis. ${ }^{7}$ The exact mechanism of myocardial injury is unknown. However, myocardial injury mediated via angiotensin-converting enzyme has been postulated ${ }^{15}$ in a murine model infected with SARS-COVID.

Hypovolemia is common in COVID-19 patients. Volume resuscitation should be based on simple bedside assessment such as pulse pressure variation, inferior vena cava collapsibility, and passive leg raising tests. ${ }^{16}$ There has been no difference in mortality with usage of colloids or crystalloids. ${ }^{17} \mathrm{~A}$ serum albumin $<3.0 \mathrm{~g} / \mathrm{dL}$ has been identified as an independent risk factor for 30-day mortality in COVID-19 patients. Thus, albumin supplementation should be considered in these patients. ${ }^{7}$

\section{Logistical Issues}

Panse et $a l^{6}$ have elegantly described the requirements to set up a COVID-19-dedicated ICU in terms of (i) ICU infrastructure capacity building, (ii) ICU staffing, (iii) ICU triage, (iv) PPE, and (v) ICU research. In the event of surging of COVID-19 patients who need ICU care and when the ICU beds are overwhelmed, patient care may be provided in a high-dependency units outside the ICU. Special ward beds may be converted into a semi-ICUs for moderately sick patients. A scheme of utilization of medical personnel as proposed by the Ministry of Health and Family Welfare, Government of India is abridged in the - Table 1. It is absolutely necessary to train medical personnel, more so if the staffing is derived from B-F categories. Attention to logistical details and maintenance of supply chains is essential to maintain all essential components of care. Innovative devices have been introduced to address the specific needs of the infection ( - Fig. 1A-D). Ethical clearance of research on COVID-19-related investigation must be done expeditiously and sharing of information through communication, online webinars, and publications becomes vital for transmission of knowledge to aid optimal patient care.

Several countries have developed mechanisms for rationing medical devices during pandemics. Rationing is performed by a triage officer or a triage committee comprising of individuals who do not have direct relationship with patient care. Triage is done in three steps: application of exclusion criteria, such as irreversible shock; assessment of mortality risk using the sequential organ failure assessment score, to determine priority for initiating ventilation; and repeat assessments over time, such that patients whose condition is not improving are removed from the ventilator to make it available for another patient. ${ }^{18}$ Triage committee should comprise of volunteers who are respected clinicians and leaders among their peers. Written recommendations attested by the government to mitigate the enormous emotional, spiritual, and existential burden to which caregivers may be exposed should be formulated by the committee. ${ }^{18}$ Triage undoubtedly raises ethical concerns, but it could be argued that these special situations need tangential thinking to make vital resources available to patients who are most likely to benefit. The key to this route of management is communication with the next of kin, empathy, involvement of medicosocial workers to subtly highlight the need of the moment. This could trigger angst among family members, but, regular counselling helps the clinical team to lead this decision. In an Indian scenario, where healthcare is not entirely free and DNAR is not justifiable, it opens a proverbial "can of worms" from a legal perspective. It is prudent to involve palliative care/endof-life care teams early in these situations. Another option is for affected patients to sign an advanced care directive that clearly should state what future quality of life they regard as acceptable and giving the clinical team an ethical base to direct therapy. It is imperative that judicious allocation of 


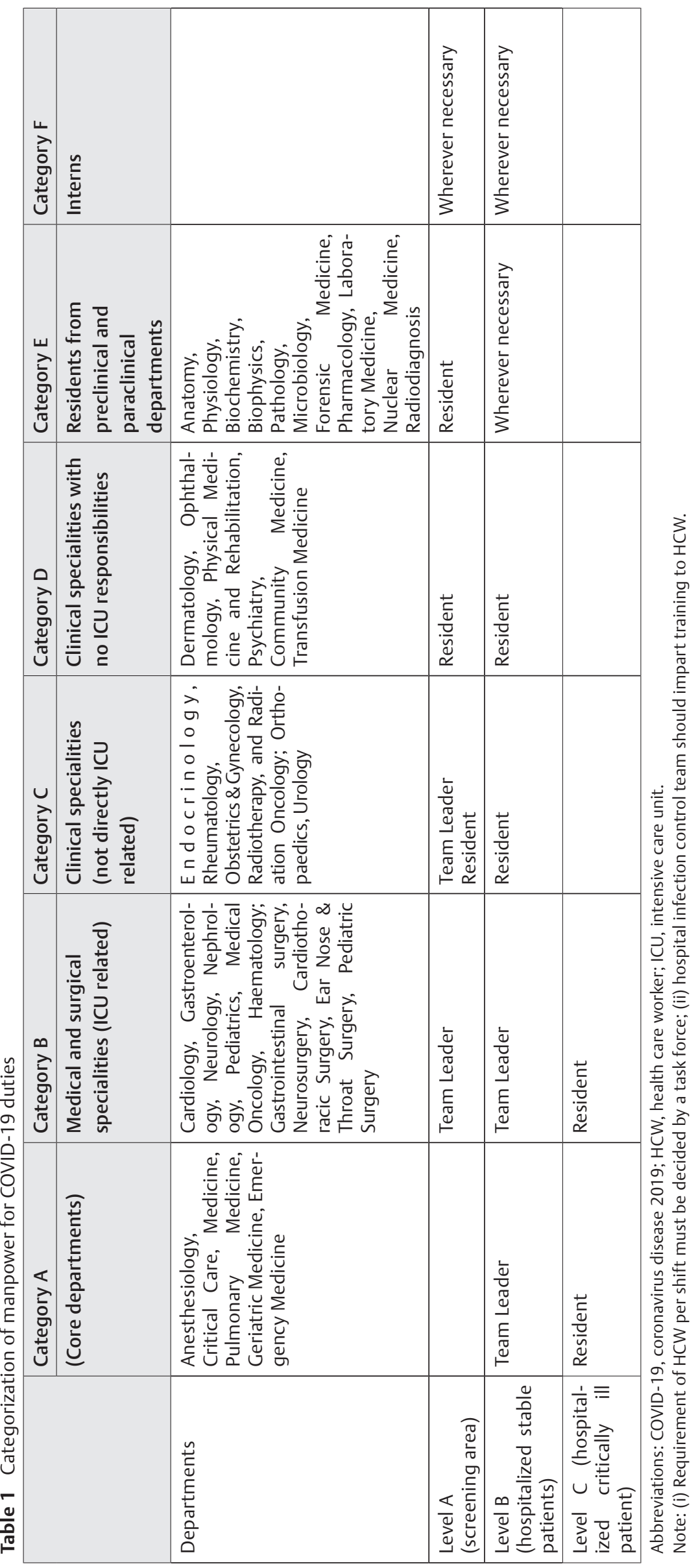




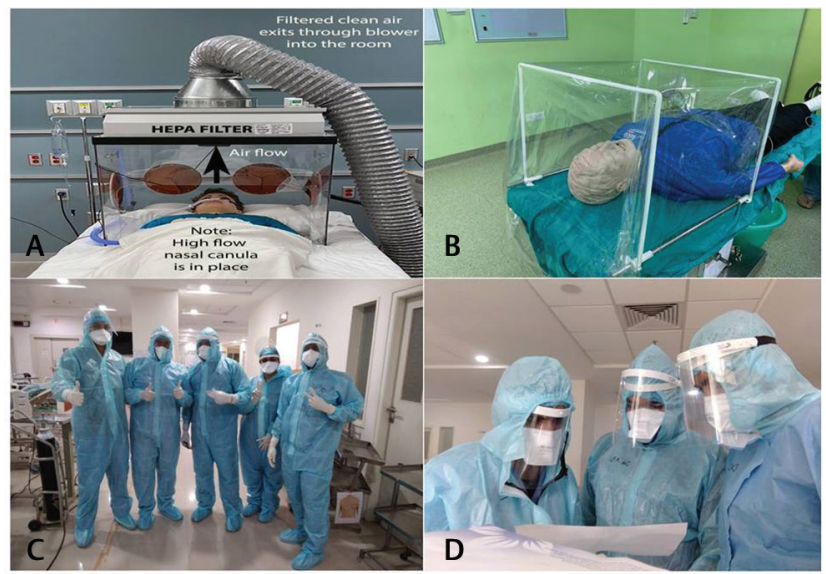

Fig. 1 Innovative devices. (A) "Aerosol hood" developed by University of Minnesota to prevent aerosol contamination of the environment during use of high-flow nasal cannula; (B) aerosol "containment" device developed by Dr Baljit Singh et al., New Delhi, with adjustable cross-bars; (C) health care worker in personal protective equipment at coronavirus disease 2019 (COVID-19) intensive care unit (ICU), Narayana Hrudayalaya (NH); (D) Intensivists at bed side rounds in a COVID-19 ICU, NH.

resources be performed in these testing times so that other non-COVID patients who deem intensive care should not be sidelined. Fair allocation and prioritization guidelines should be developed to enable clinical teams make evidence-based decisions without emotional burden.

HCW should be segregated to reduce risk of cross-transmission that allows for a safe and sustainable service should a group be quarantined. The HCW should only be designated to particular patient care with well-defined geographical areas. In the event of widespread community transmission, the ICU manpower can be seriously depleted. The patient to HCW ratio should be maintained as per institutional policies. High pressure working environments can increase anxiety levels causing significant physical and mental fatigue. Open channels of communication and dissemination of latest guidelines along with psychological support can mitigate these issues. ${ }^{19} \mathrm{~A}$ productive strategy is to incorporate counseling services for $\mathrm{HCW}$ to ensure mental well-being. ${ }^{20}$

To conclude, we need to remember nothing lasts forever and these testing times will pass eventually. We need to put our resources to good use and focus on delivering a sustainable level of care. Once the disease process is well understood and logistics catered to, the disease tide will ebb. Quoting CJ Redwine-"Losing your head in a crisis is a good way to become a crisis."

\section{Conflict of Interest}

None.

\section{References}

1 Huang C, Wang Y, Li X, et al. Clinical features of patients infected with 2019 novel coronavirus in Wuhan, China. Lancet 2020;395(10223) :497-506

2 Liew MF, Siow WT, MacLaren G, See KC. Preparing for COVID-19: early experience from an intensive care unit in Singapore. Crit Care 2020;24(1):8310.1186/s13054-020-2814-X
3 Maves RC, Jamros CM, Smith AG. Intensive care unit preparedness during pandemics and other biological threats. Crit Care Clin 2019;35(4):609-618

4 Cheng VC, Chan JF, To KK, Yuen KY. Clinical management and infection control of SARS: lessons learned. Antiviral Res 2013;100(2):407-419

5 Edelson DP, Sasson C, Chan PS, et al; American Heart Association ECC Interim COVID Guidance Authors. Interim guidance for basic and advanced life support in adults, children, and neonates with suspected or confirmed COVID-19: from the Emergency Cardiovascular Care Committee and get with the guidelines-resuscitation adult and pediatric task forces of the American Heart Association. Circulation 2020;141(25):e933-e943

6 Panse S, Kanchi M, Chacko J, et al. Intensive care until setup for COVID-19. J Card Crit Care. 2020;00:1-7

7 Shang Y, Pan C, Yang X, et al. Management of critically ill patients with COVID-19 in ICU: statement from front-line intensive care experts in Wuhan, China. Ann Intensive Care 2020;10(1):7310.1186/s13613-020-00689-1

8 Xu Y, Meng M, Li L, et al. Recommendations for bronchoscopy procedures in severe COVID-19 patients. Chin J. Crit. Care Med. 2020;6:E031

9 Arabi YM, Fowler R, Hayden FG. Critical care management of adults with community-acquired severe respiratory viral infection. Intensive Care Med 2020;46(2):315-328

10 Wang YM, Zhang DY, Du GH, et al. Remdesivir in adults with severe COVID-19: a randomized, double-blind, placebocontrolled, multicenter trial. Lancet 2020;395(10236): 1569-1578

11 Yang X, Yang Q Wang Y, et al. Thrombocytopenia and its association with mortality in patients with COVID-19. J Thromb Haemost 2020;18(6):1469-1472

12 Tang N, Bai H, Chen X, Gong J, Li D, Sun Z. Anticoagulant treatment is associated with decreased mortality in severe coronavirus disease 2019 patients with coagulopathy. J Thromb Haemost 2020;18(5):1094-1099

13 Chen Y, Luo R, Wang K, et al. Kidney Impairment Is Associated with In-Hospital Death of COVID-19 Patients. MedRxiv; 2020Accessed Aug 21, 2020

14 Zhang JY, Shao $\mathrm{CH}$, Yang JH, et al. Recommendations for Nutrition Therapy in Critically Ill COVID-19 Patients. Chin J Clin Med. 2020. http://kns.cnki.net/kcms/detail/31.1794. R.20200311.0958.002.html. Accessed Aug 21, 2020

15 Mancia G, Rea F, Ludergnani M, et al. Angiotensin-Aldosterone System Blockers and the Risk of COVID-19. N Engl J Med. 2020. https://doi.or/10.1056/NEJMoa2006923. Accessed Aug 21, 2020

16 Yang X, Du B. Does pulse pressure variation predict fluid responsiveness in critically ill patients? A systematic review and meta-analysis. Crit Care 2014;18(6):650

17 Lewis SR, Pritchard MW, Evans DJ, et al. Colloids versus crystalloids for fluid resuscitation in critically ill people. Cochrane Database Syst Rev 2018;8:CD000567

18 Truog RD, Mitchell C, Daley GQ. The toughest triage - allocating ventilators in a pandemic. N Engl J Med 2020; 382(21):1973-1975

19 Goh KJ, Wong J, Tien JC, et al. Preparing your intensive care unit for the COVID-19 pandemic: practical considerations and strategies. Crit Care 2020;24(1):21510.1186/s13054-020-02916-4

20 Ehrlich H, McKenney M, Elkbuli A. Protecting our healthcare workers during the COVID-19 pandemic. Am J Emerg Med 2020;38(7):1527-1528 